\title{
Systematic development of predictive mathematical models for animal cell cultures
}

Cleo Kontoravdi ${ }^{1}$, Efstratios N. Pistikopoulos ${ }^{1}$ and Athanasios Mantalaris ${ }^{1, *}$

${ }^{1}$ Biological Systems Engineering Laboratory, Centre for Process Systems Engineering, Department of Chemical Engineering, Imperial College London, South Kensington Campus, London SW7 2AZ,

*Corresponding Author Biological Systems Engineering Laboratory

Centre for Process Systems Engineering

Department of Chemical Engineering

Imperial College London

South Kensington campus

London SW7 2AZ

Phone: +44(0)2075945601

Fax: +44(0)2075945638

Email: a.mantalaris@imperial.ac.uk 


\begin{abstract}
Fed-batch cultures are used in producing monoclonal antibodies industrially. Existing protocols are developed empirically. Model-based tools aiming to improve productivity are useful with model reliability and computational demand being important. Herein, a systematic framework for developing predictive models is presented comprising of model development, global sensitivity analysis, optimal experimental design for parameter estimation, and predictive capability checking. Its efficacy and validity are demonstrated using a fed-batch structured/unstructured model of antibody-secreting hybridoma cultures. Global sensitivity analysis is first used to identify sensitive model parameters (initial values estimated from batch cultures). Information-rich data from an optimally designed fed-batch experiment are then used to estimate these parameters, resulting in good agreement between simulation and experimental results. Finally, the model's predictive capability is confirmed by comparison with an independent set of fed-batch cultures. This approach systematises the process of developing predictive cell culture models at a minimum experimental cost, enabling modelbased control and optimisation.
\end{abstract}




\section{INTRODUCTION}

Increasing demand for monoclonal antibodies (MAbs), where time to market becomes critical, could benefit from the use of model-based techniques with the aim of optimising and controlling cell viability and productivity. The development of reliable mathematical models of animal cell cultures is, of course, critical for such applications. In general, these models can be classified as either structured or unstructured (Bibila and Robinson, 1995). Unstructured models do not take into account the inner structure of the cell, while structured models incorporate biological knowledge by lumping the biomaterial into distinct compartments (Sidoli et al., 2004). Even though structured models constitute a more detailed and biologically consistent representation of cellular activities, they examine single cells and fail to describe the collective behaviour of the entire population. Attempts to overcome this limitation, including the combination of such single-cell models with population balance equations (Sidoli et al., 2006) or simulation of a large number of single-cell models with different initial conditions (Domach and Shuler, 1984), pose significant computational and validation challenges that render them unsuitable for further application.

Unstructured models, on the other hand, have been proposed as an appropriate basis for applying control, optimization and process development techniques to the production process of proteins (Dowd et al., 1999; Dhir et al., 2000)). Their main advantages are that they involve extracellular culture variables that are typically monitored during a culture, as well as that their simulation and subsequent in silico applications are computationally tractable. One of the major problems associated with unstructured models though is their limited applicability to the process conditions and data range they are derived from. However, it has been put forward that the growth of a cell line follows the same kinetics, irrespective of the cultivation mode (Pörtner and Schäfer, 1996). It is, therefore, possible that an unstructured model developed from batch culture data can also describe fed-batch cultures, which are known to increase levels of protein production and are preferred industrially, as long as it is properly validated.

For structurally correct models, validation pertains to successful parameter estimation. In the case of cell culture models, estimation is usually carried out for all model parameters and using already existing (most often, batch) data. Nevertheless, not all parameters may be identifiable or estimable, hence the need for an identifiability analysis, or, in the case of dynamic, nonlinear models, a sensitivity analysis. The latter studies how a variation in the model output can be apportioned to the variation of the different parameters. It is therefore used to assess which reduced set of parameters needs to be estimated from experimental data 
in order to increase model precision. However, unique estimation of the parameter set is only possible if the available data are sufficiently rich (Versyck et al., 1997). The use of optimal experimental design for estimation of the values of 'sensitive' parameters can result in significant improvements in parameter confidences (Versyck et al., 1997; Nathanson and Saidel, 1985; Munack and Posten, 1989). Optimal experimental design targets the determination of input profiles that generate informative experimental data, which then enable accurate parameter estimation (Munack, 1989; Munack and Posten, 1989). Optimal experimental design can therefore aid the development of a predictive model applicable to a wider range of culture conditions through the design of highly dynamic fed-batch experiments. Hence, it is proposed to employ these model-based tools in order to systematise the methodology for developing predictive mathematical models of protein-producing animal cell cultures. The framework put forward and validated herein consists of four steps, namely model development, global sensitivity analysis, optimal experimental design for parameter estimation, and predictive capability checking, as outlined in Figure 1.

In a previous study (Kontoravdi et al., 2005), a hybrid model of antibody-producing mammalian cell cultures was presented and compared to experimental data for the hybridoma 14-4-4S cell line (Tatiraju et al., 1999). The model is based on the assumption that cell growth depends on the availability of two key nutrients, glucose and glutamine, and the accumulation of two toxic metabolites, lactate and ammonia. Cell death depends on the accumulation of ammonia due to glutamine metabolism and spontaneous degradation in the medium. The synthesis of IgG1 antibody product is described starting from the gene copy number and synthesis of heavy- and light-chain mRNA molecules. These are then translated into heavy and light antibody chains, which sequentially combine to form the antibody molecule (two heavy and two light chains) in the endoplasmic reticulum. This molecule then travels to the Golgi apparatus and is finally secreted into the extracellular medium. The model equations are listed in appendix A and consist of 28 differential and algebraic equations containing 30 parameters. In summary, the model describes cell growth and antibody synthesis and production in the context of laboratory-scale cell cultures. The Sobol' method of global sensitivity analysis was used to identify the model parameters to which the model output (MAb concentration) was most sensitive, prompting the need for their accurate estimation from experimental data.

In this work, optimal experimental design tools are applied to the aforementioned model in order to successfully estimate the values of the 'sensitive' parameters and extend the range of the model's validity to fed-batch conditions. First, a supplementary one-at-a-time screening of parameter properties with respect to all measured variables was carried out to identify the 
targets of the parameter estimation and experimental design, thus complementing the previous global sensitivity analysis. Batch culture experiments of IgG1-secreting hybridoma cells were then performed to provide initial estimates for those parameters, since parameter values relating to cell growth and product synthesis and secretion are cell line-specific. Following that, D-optimal dynamic experiments were designed to improve parameter accuracy and the validity of the resulting model under fed-batch conditions was confirmed by comparison to an independent set of fed-batch culture data. To our knowledge, this is one of the first attempts to formalise the model development process for cell culture systems, coupling experimentation and model-based tools at each step. 


\section{MATERIALS AND METHODS}

Hybridoma cell line HFN7.1 producing IgG1 against fibronectin from human plasma (CRL1606 from ATCC) was cultured in high-glucose (4.5g/L) DMEM (Gibco) supplemented with $2.5 \%$ bovine calf serum (ATCC). Additional glucose and glutamine required for the fed-batch cultures were obtained from Sigma-Aldrich. Both batch and fed-batch cultures were conducted in 1L Erlenmeyer flasks with a working volume of $200 \mathrm{ml}$ and inoculation cell density of $2 \times 10^{8} \mathrm{cells} / \mathrm{L}$. Temperature and $\mathrm{CO}_{2}$ air concentration were automatically controlled at $37^{\circ} \mathrm{C}$ and $5 \%$, respectively. Cell density was measured using a hemacytometer, where cell viability was determined by the method of Trypan Blue dye exclusion. For determination of nutrient, metabolite and antibody concentration in the culture supernatant, $1.5 \mathrm{~mL}$ samples were withdrawn from the culture, the cells centrifuged at $10^{4} \mathrm{~g}$ for 5 minutes in an Eppendorf microfuge, and the supernatants stored at $-20{ }^{\circ} \mathrm{C}$. Extracellular glucose, glutamine, lactate and ammonia concentrations were measured with the YSI Bioprofiler 200 (Nova Biomedical, U.K.).

Antibody concentration in the supernatant was estimated by indirect sandwich enzyme-linked immunosorbent assay (ELISA). Specifically, non-cell culture treated 96-well plates (Sigma, UK) were coated with $100 \mu \mathrm{L}$ of $1 \mu \mathrm{g} / \mathrm{ml}$ anti-human fibronectin antibody from rabbit (Sigma) in coating buffer (0.05 M sodium bicarbonate, $\mathrm{pH}$ 9.6) after an overnight incubation at $4{ }^{\circ} \mathrm{C}$. The plates were blocked for non-specific binding with $250 \mu \mathrm{L} /$ well of blocking buffer (250 ml coating buffer with $1.25 \mathrm{~g}$ cassein hammerstein; Sigma) for $1 \mathrm{hr}$ at room temperature. The plates were washed with washing buffer (phosphate buffered saline with $0.05 \%$ Tween). Subsequently, $100 \mu \mathrm{L}$ of the sample or the control $(1 \mathrm{mg} / \mathrm{ml}$ human fibronectin diluted in PBS at a ratio of 1:5,000; Chemicon, UK) were added per well and incubated for $1 \mathrm{hr}$ on an orbital plate shaker at room temperature. The plates were washed as before followed by addition of $100 \mu \mathrm{L} /$ well of mouse anti-human fibronectin antibody from mouse (Sigma) diluted in PBS at a ratio of 1:5,000 and incubated for 2 hrs at room temperature on an orbital plate shaker. The plates were washed as before and $100 \mu \mathrm{L}$ of 6.4 $\mathrm{mg} / \mathrm{ml}$ anti-mouse $\mathrm{Fc}$ antibody from goat (Sigma) diluted in PBS at a ratio of 1:10,000 and incubated for $1 \mathrm{hr}$ at room temperature on an orbital plate shaker. Subsequently, the plates were washed followed by visualisation of the reaction using the 3,3',5,5'-tetramethylbenzene kit (TMB; Sigma-Aldrich T3405) as per the manufacturer's instructions. The absorbance was read at $450 \mathrm{~nm}$ on a microplate reader (BioTek Instruments, Inc., USA).

Batch cultures were carried out in triplicate flasks using the aforementioned DMEM medium, which corresponds to initial concentrations of $29.1 \mathrm{mM}$ for glucose and $4.9 \mathrm{mM}$ for glutamine, 
and $2.5 \%$ bovine calf serum. Fed-batch cultures were carried out in duplicate flasks using two different sets of initial concentrations. In the first fed-batch culture experiment used for model validation, the initial concentration of glucose was $25.1 \mathrm{mM}$ and of glutamine $5.01 \mathrm{mM}$. In the second, independent experiment, the initial concentration of glucose was $18.4 \mathrm{mM}$ and of glutamine $3.74 \mathrm{mM}$. The same concentrated medium was supplied to both sets of fed-batch cultures in pulses and the concentrations of glucose and glutamine in the feed were set at $500 \mathrm{mM}$ and $100 \mathrm{mM}$, respectively. The maximum total volume of feed was fixed at $8.75 \mathrm{ml}$, which represents less than $5 \%$ of the total culture volume $(200 \mathrm{ml})$, so as to avoid dilution effects. 


\section{MODEL DEVELOPMENT PLATFORM}

\subsection{Model analysis}

The Sobol' method of global sensitivity analysis was employed to quantitatively assess the effect of variation in model parameter values on model output, in this case MAb concentration. This is a variance-based Monte-Carlo method that examines the entire range of parameter values based on the ANOVA (Analysis of Variances) decomposition of a given function into summands of increasing dimensionality (Sobol', 2001). A further brief one-atat-a-time screening of model parameters was carried out for evaluating to which parameters the remaining experimentally-measured outputs are sensitive. Hence, the effect of all parameters on viable and total cell concentrations was investigated based on model simulation.

The global sensitivity analysis identified 8 parameters to which MAb concentration was most sensitive (Kontoravdi et al., 2005). A further one-at-a-time screening was performed to determine the parameters by which the measured variables were most influenced, since a full map of sensitivity indices with respect to all measurements was not available. Those were found to be the yield of cells on glucose $\left(\mathrm{Y}_{\mathrm{x}, \mathrm{glc}}\right)$, the yield of cells on glutamine $\left(\mathrm{Y}_{\mathrm{x}, \mathrm{gln}}\right)$, the yield of lactate on glucose $\left(\mathrm{Y}_{\text {lac,glc }}\right)$, the yield of ammonia on glutamine $\left(\mathrm{Y}_{\mathrm{amm}, \mathrm{gln}}\right)$, the rate of dead cell lysis $\left(\mathrm{K}_{\mathrm{lysis}}\right)$, the maximum specific cell growth rate $\left(\mu_{\max }\right)$, and the gene copy number of MAb heavy chains $\left(\mathrm{N}_{\mathrm{H}}\right)$. These parameters were targeted for the parameter estimation process, whereas certain parameters identified through the global sensitivity analysis study were set at their nominal values as they involved intracellular measurement or cell cycle analysis that were not carried out in this study.

Batch culture experiments were initially carried out, through which the validity of the model structure to the HFN7.1 hybridoma cell line was confirmed (simulations were carried out using gPROMS (PSE, 2002a)). As shown in Figure 2, the model captures the trend of viable cell concentration during the initial lag phase and correctly predicts the height and time of the peak in the number of viable cells. Model results predict higher concentrations during the exponential growth phase, but the overall experimental trend is observed. Similarly, during the decline of the viable cell concentration the model correctly captures the shape of the concentration profile and, in most cases, provides an accurate prediction of the viable cell number. Moreover, MAb concentration is accurately predicted throughout the duration of the culture (Figure 2). Similar agreement was achieved for glutamine and ammonia (Figure 3), and glucose and lactate (Figure 4). 


\subsection{Optimal experimental design and parameter estimation}

The accurate estimation of the aforementioned 7 parameters under fed-batch conditions was the aim of the optimal experimental design, which was conducted using the initial estimates as determined from the batch culture data (Table 1). D-optimal experimental design was used to maximise the information content of experimental data, specifically for the estimation of the seven parameters identified in the sensitivity analysis and one-at-atime screening. This is done by minimising the volume of the confidence ellipsoid, i.e., minimising the determinant of the variance-covariance matrix, $\mathrm{V}$, of the parameters to be estimated.

The optimisation problem seeks to determine the initial conditions, experiment duration, variation of controls and timing of samples such that the maximum amount of information is generated with the given measurements. These measurements were nutrient and metabolite concentrations, the viable and total cell concentrations and the extracellular MAb concentration. The design was conducted in the gPROMS (PSE, 2002b) modelling environment, which has a dedicated function, namely 'experiment design for parameter precision', and uses a SRQPD sequential quadratic programming code. The amount of feed, feeding interval, and experiment duration were treated as degrees of freedom within certain operational limits. The initial glucose concentration was limited between 5.5 and $25 \mathrm{mM}$. The feed was allowed to vary between 0 and $12.5 \mathrm{ml}$ per hour. Concentrated medium was supplied to the culture vessel in pulses and the maximum total volume of feed was fixed at $8.75 \mathrm{ml}$, which represented less than $5 \%$ of the total culture volume $(200 \mathrm{ml})$, so as to avoid dilution effects. The amount of feed supplied at each feeding interval was optimised by the design, as was the timing of these intervals. The earliest measurement time was set at 12 hours and the minimum time between measurements at 6 hours. The duration of the experiment was allowed to vary between 4 and 8 days. The optimal duration was determined at 168 hours (7 days), during which a pulse feed was introduced once a day, at an interval of 24 hours.

The sequence of designing an experiment, performing it, and using the generated data for model validation is done iteratively, until model predictions and experimental data are in satisfactory agreement. The results of the designed experiments were used to estimate the values of model parameters using the 'parameter estimation' entity in gPROMS based on the same SRQPD sequential quadratic programming code. Parameter 
estimation was based on the maximum likelihood formulation, which attempts to determine values for the uncertain physical and variance model parameters that maximise the probability that the model will predict the measurement values obtained from the experiments. The statistical variance model of constant variance was used in this case. In this case, the validation process was successful after one fed-batch experiment as the $95 \%$ confidence intervals were deemed satisfactory at $\pm 10 \%$ of the final parameter values.

The complete set of parameter values for fed-batch cultures can be seen in Table 1, where they are compared to the corresponding values for batch culture operation. The major differences are in the parameters describing nutrient utilisation, as expected. Increased uptake of both nutrients in fed-batch culture also results in prolonged cell growth and higher viable (and hence, total) cell concentration, and is reflected in the higher values in cell yield on glucose and glutamine $\left(2.6 \times 10^{8}\right.$ and $8 \times 10^{8}$, respectively) compared to values for batch culture $\left(1.1 \times 10^{8}\right.$ and $5.6 \times 10^{8}$, respectively). The yield of lactate on glucose is at its maximum value of 2 in the case of fed-batch culture, while it is 1.4 in batch culture. This is due to the increased availability and utilisation of glucose, which leads to higher conversion into lactate, and agrees with previous experimental observations (Glacken et al., 1986; Miller et al., 1988). Finally, there is some difference in the values of the heavy and light chain gene copy number. As the values for batch cultures represent initial estimates, these are estimated using the data for fed-batch operation. The estimated value of 100 genes per cell for both the heavy and the light chain is therefore considered to be the most accurate estimate since these figures should be independent of culture operation mode.

The results of the parameter estimation and the uncertainty in parameter values expressed in confidence intervals are shown in Table 2. The confidence intervals of the estimated parameters are satisfactory, with the $95 \%$ confidence intervals falling within $\pm 10 \%$ of the final value for all parameters except $\mathrm{Y}_{\mathrm{amm}, \mathrm{gln}}( \pm 17 \%)$. The resulting model is in good agreement with the results of this first experiment (Figures 5 to 7). More specifically, predictions and experimental data for viable and total cell concentrations can be seen in Figure 5. Simulation results for viable cell concentration closely match the data during the first 60 hours. Thereafter, model predictions follow the trend of the experimental data correctly predicting the plateau in viable cell concentration. Over the last 40 hours the model over-predicts the concentration of viable cells in the culture. This is probably because the viable cell concentration in vitro is too low for the cells to recover viability, but the model predicts that will occur as more concentrated feed is supplied to the culture. The data for total 
cell concentration are more accurately matched by the simulation results (Figure 5). Again, there is good agreement over the first 100 hours, with few discrepancies thereafter. The overall trend of the concentration is correctly captured by the model.

Figure 6 shows good agreement between model simulation results and experimental data for glutamine and ammonia. Glutamine concentration is correctly predicted throughout the duration of the culture. Discrepancies only occur once glutamine has reached zero concentration (after 80 hours), when additional glutamine fed to the culture is metabolised more quickly than predicted. Ammonia concentration is also closely tracked by model results. It is correctly predicted during the initial lag phase, over-predicted over the following 70 hours, and closely matched during the remainder of the culture. Finally, model simulation results for extracellular MAb concentration are in good agreement with experimental data as shown in Figure 7. The model closely tracks the data throughout the duration of the culture, correctly predicting the MAb concentration during the lag and exponential growth phases, as well as the final concentration in the medium. Overall, the model captures the profiles of all measure variables successfully.

\subsection{Predictive capability demonstration}

In order to demonstrate the model's predictive capability, its simulation results were compared with an independent set of data from a fed-batch experiment. The initial glucose and glutamine concentrations were lower than in the designed experiment and the same amount of concentrated feed was introduced in two instead of one dose per day (once every 12 hours). The sampling schedule was the same as in the designed experiment, with viable and total cell, nutrient, metabolite, and extracellular MAb concentrations determined at each point. Glutamine and ammonia concentrations are accurately predicted by the model as shown in Figure 8. Simulation results closely track the experimental data for glutamine, with some discrepancies between 60 and 90 hours, when the predictions are marginally lower than the experimental values. Ammonia concentration data are also matched by model results. There are some small discrepancies between 20 and 70 hours, when the predicted concentration is higher than observed, and during the final few hours of the culture, when the concentration is marginally under-predicted.

In terms of the cell concentrations, the trends of both viable and total cell profiles are predicted satisfactorily. The model closely tracks the data for viable cell concentration during the first 70 hours of the culture, correctly predicting the lag and exponential growth phases (Figure 9). The in silico peak in concentration is lower than the in vitro, but subsequent predictions match the data. As in the case of the designed fed-batch experiment, there are 
discrepancies in the final few hours of the culture, when the model predicts that the cells can recover their viability since more nutrients are supplied, but that is not observed experimentally. The total cell concentration is correctly predicted throughout the culture. There is good agreement between simulation results and experimental data during the lag and exponential growth phases. The peak in concentration is then predicted to be higher than that observed, after which the decline in cell number is closely tracked by the model. Finally, the final extracellular concentration of monoclonal antibodies is correctly estimated by the model (Figure 10). This concentration rises more sharply than calculated by the model during the exponential growth phase, however, the final concentration achieved is successfully calculated.

Overall, based on the level of biological information that is described by the existing model structure, the agreement between simulation results and experimental data from the independent fed-batch experiment is satisfactory. Previous applications of optimal experimental design in this field were restricted to simpler systems of microorganisms (Bernaerts et al., 2000) and were often incomplete, as parameter estimation was performed using simulated results (Versyck et al., 1997). To our knowledge, this is one of the first attempts to close the loop between model-based tools and designed experimentation in biological systems and to formalise the steps of the model development process for animal cell cultures, avoiding trial-and-error practices and, thus, unnecessary experimentation. The resulting model, which was developed at a minimum experimental cost, can be used for in silico experimentation, as well as model-based control and optimisation studies. 


\section{CONCLUDING REMARKS}

A systematic framework for model analysis and experimental design was presented, which was used to improve the reliability of a simple, hybrid model of animal cell cultures and to extend its range of applicability from batch to fed-batch culture conditions. Overall, the framework presented and validated here proposes a well-defined step-by-step methodology for building predictive models of cell culture processes at a minimum experimental cost, while avoiding trial-and-error practices. This systematic combination of modelling and experimentation through process systems engineering tools can, in the future, guide process development in the biopharmaceutical industry with an aim to reduce the time required for a product to reach the market.

ACKNOWLEDGEMENTS. CK thanks the Alexander S. Onassis Public Benefit Foundation for her fellowship. Financial support from the Centre for Process Systems Engineering and its Industrial Consortium is also acknowledged. 


\section{NOTATION}

$\mathrm{K}$

heavy- and light-chain mRNA decay rate $\left(\mathrm{h}^{-1}\right)$

$\mathrm{K}_{\mathrm{A}} \quad$ assembly rate constant (cell $/$ molecule $/ \mathrm{h}$ )

$\mathrm{K}_{\mathrm{d}, \mathrm{amm}} \quad$ ammonia constant for cell death $(\mathrm{mM})$

$\mathrm{K}_{\mathrm{d}, \mathrm{gln}} \quad$ constant for glutamine degradation $\left(\mathrm{h}^{-1}\right)$

$\mathrm{K}_{\mathrm{ER}} \quad$ rate constant for ER-to-Golgi transport $\left(\mathrm{h}^{-1}\right)$

$\mathrm{K}_{\mathrm{G}} \quad$ rate constants for Golgi-to-medium antibody transport $\left(\mathrm{h}^{-1}\right)$

$\mathrm{K}_{\mathrm{glc}} \quad$ Monod constant for glucose $(\mathrm{mM})$

$\mathrm{K}_{\mathrm{gln}} \quad$ Monod constant for glutamine (mM)

$\mathrm{KI}_{\mathrm{amm}} \quad$ Monod constant for ammonia (mM)

$\mathrm{KI}_{\text {lac }} \quad$ Monod constant for lactate $(\mathrm{mM})$

$\mathrm{m}_{\text {glc }} \quad$ maintenance coefficient of glucose $(\mathrm{mmol} / \mathrm{cell} / \mathrm{h})$

$\mathrm{N}_{\mathrm{H}}, \mathrm{N}_{\mathrm{L}} \quad$ heavy- and light-chain gene copy number (gene/cell)

$\mathrm{R}_{\mathrm{H}}, \mathrm{R}_{\mathrm{L}} \quad$ rates of heavy- and light-chain consumption in assembly (chain/cell/h)

$\mathrm{S}_{\mathrm{H}}, \mathrm{S}_{\mathrm{L}} \quad$ heavy- and light-chain gene specific transcription rates (mRNA/gene/h)

$\mathrm{T}_{\mathrm{H}}, \mathrm{T}_{\mathrm{L}} \quad$ heavy- and light-chain specific translation rates (chain/mRNA/h)

$\mathrm{Y}_{\mathrm{amm}, \mathrm{gln}}$ yield of ammonia from glutamine $(\mathrm{mmol} / \mathrm{mmol})$

$\mathrm{Y}_{\text {lac,glc }} \quad$ yield of lactate from glucose $(\mathrm{mmol} / \mathrm{mmol})$

$\mathrm{Y}_{\mathrm{x}, \mathrm{glc}} \quad$ yield of cells on glucose $(\mathrm{cell} / \mathrm{mmol})$

$\mathrm{Y}_{\mathrm{x}, \mathrm{gln}} \quad$ yield of cells on glutamine (cell $\left./ \mathrm{mmol}\right)$

\section{Greek letters}

$\alpha_{1}, \alpha_{2} \quad$ constants of glutamine maintenance coefficient $(\mathrm{mM} \mathrm{L} / \mathrm{cell} / \mathrm{h}$ and $\mathrm{mM}$, respectively)

$\gamma_{1} \quad$ constant for antibody production (h)

$\gamma_{2} \quad$ constant for antibody production (dimensionless)

$\varepsilon_{1} \quad$ ER glycosylation efficiency factor (dimensionless)

$\varepsilon_{2} \quad$ Golgi apparatus glycosylation efficiency factor (dimensionless)

$\mu_{\mathrm{dmax}} \quad$ maximum specific death rate $\left(\mathrm{h}^{-1}\right)$

$\mu_{\max } \quad$ maximum specific growth rate $\left(\mathrm{h}^{-1}\right)$ 


\section{REFERENCES}

1. Bibila T. A. and Robinson D. K. (1995) Biotechnol. Prog. 11, 1-13.

2. Dhir S., Morrow K. J., Rhinehart R. R. \& Wiesner T (2000) Biotechnol. Bioeng. 67, 197205.

3. Domach M. M. and Shuler M. L. (1984) Biotechnol. Bioeng. 26, 877-884.

4. Dowd J. E., Weber I., Rodriguez B., Piret J. M. and Kwok K. E. (1999) Biotechnol. Bioeng. 63, 484-492.

5. Glacken M.W., Fleischaker R.J. and Sinskey A.J. (1986). Biotechnol. Bioeng. 28, 13761389.

6. Kontoravdi C., Asprey S. P., Pistikopoulos E. N. and Mantalaris A. (2005) Biotechnol. Prog. 21, 1128-1135.

7. Miller W.M., Blanch H.W. and Wilke C.R. (1988). Biotechnol. Bioeng. 32, 947-965.

8. Munack A. (1989) in Computer Applications in Fermentation Technology Modelling and Control of Biotechnological Processes (Elsevier).

9. Munack A. and C. Posten C. (1989) Proc. Am. Control Conference ACC89, Pittsburgh, PA 2011-2016.

10. Nathanson M. H. and Saidel G. M. (1985) Am. J Physiol. 248, R378-R386.

11. Pörtner R. and Schäfer T. (1996) J Biotechnol. 49, 119-135.

12. Process Systems Enterprise (2002a) gPROMS Introductory User Guide (U.K.).

13. Process Systems Enterprise (2002b) gPROMS Advanced User Guide (U.K.).

14. Sidoli F. R., Asprey S. P. and Mantalaris A. Ind. Eng. Chem. Res. 45, 5801-5811.

15. Sobol' IM (2001). Mathematics and Computers in Simulation 55, 271-280.

16. Tatiraju S. Soroush M. and Mutharasan R. (1999) Biotechnol. Bioeng. 63, 22-32.

17. Sidoli F, Mantalaris A, Asprey (2004) Cytotechnology 44(1-2), 27-46.

18. Versyck K. J., Claes J. E. and Van Impe J. F. (1997) Biotechnol. Prog. 13, 524-531. 


\section{TABLES}

Table 1. Parameter values for batch and fed-batch culture operations.

\begin{tabular}{|c|c|c|}
\hline Parameter & Batch operation value & Fed-batch operation value \\
\hline $\mathrm{K}\left(\mathrm{h}^{-1}\right)$ & $10^{-1}$ & $10^{-1}$ \\
\hline $\mathrm{K}_{\mathrm{A}}($ cell $/$ molecule- $\mathrm{L})$ & $10^{-6}$ & $10^{-6}$ \\
\hline $\mathrm{K}_{\mathrm{d}, \mathrm{amm}}(\mathrm{mM})$ & 1.76 & 1.76 \\
\hline $\mathrm{K}_{\mathrm{d}, \mathrm{gln}}\left(\mathrm{h}^{-1}\right)$ & $9.6 \times 10^{-3}$ & $9.6 \times 10^{-3}$ \\
\hline $\mathrm{K}_{\mathrm{ER}}\left(\mathrm{h}^{-1}\right)$ & $6.9 \times 10^{-1}$ & $6.9 \times 10^{-1}$ \\
\hline $\mathrm{K}_{\mathrm{G}}\left(\mathrm{h}^{-1}\right)$ & $1.4 \times 10^{-1}$ & $1.4 \times 10^{-1}$ \\
\hline $\mathrm{K}_{\mathrm{glc}}(\mathrm{mM})$ & $7.5 \times 10^{-1}$ & $7.5 \times 10^{-1}$ \\
\hline $\mathrm{K}_{\mathrm{gln}}(\mathrm{mM})$ & $7.5 \times 10^{-2}$ & $7.5 \times 10^{-2}$ \\
\hline $\mathrm{KI}_{\mathrm{amm}}(\mathrm{mM})$ & 28.48 & 28.48 \\
\hline $\mathrm{KI}_{\mathrm{lac}}(\mathrm{mM})$ & 171.76 & 171.76 \\
\hline $\mathrm{K}_{\text {lysis }}\left(\mathrm{h}^{-1}\right)$ & $5.5 \times 10^{-2}$ & $3.0 \times 10^{-2}$ \\
\hline $\mathrm{m}_{\mathrm{glc}}(\mathrm{mmol} / \mathrm{cell}-\mathrm{h})$ & $4.9 \times 10^{-14}$ & $4.9 \times 10^{-14}$ \\
\hline $\mathrm{n}(-)$ & 2 & 2 \\
\hline $\mathrm{N}_{\mathrm{H}}($ gene/cell $)$ & $1.4 \times 10^{2}$ & $1.0 \times 10^{2}$ \\
\hline $\mathrm{N}_{\mathrm{L}}$ (gene/cell) & $1.2 \times 10^{2}$ & $1.0 \times 10^{2}$ \\
\hline $\mathrm{S}_{\mathrm{H}}(\mathrm{mRNA} / \mathrm{gene}-\mathrm{h})$ & $3 \times 10^{3}$ & $3 \times 10^{3}$ \\
\hline $\mathrm{S}_{\mathrm{L}}(\mathrm{mRNA} /$ gene-h) & $4.5 \times 10^{3}$ & $4.5 \times 10^{3}$ \\
\hline $\mathrm{T}_{\mathrm{H}}$ (chain/mRNA-h) & 17 & 17 \\
\hline $\mathrm{T}_{\mathrm{L}}$ (chain/mRNA-h) & 11.5 & 11.5 \\
\hline $\mathrm{Y}_{\mathrm{amm}, \mathrm{gln}}(\mathrm{mmol} / \mathrm{mmol})$ & $4.3 \times 10^{-1}$ & $4.5 \times 10^{-1}$ \\
\hline $\mathrm{Y}_{\mathrm{lac}, \mathrm{glc}}(\mathrm{mmol} / \mathrm{mmol})$ & 1.4 & 2.0 \\
\hline $\mathrm{Y}_{\mathrm{x}, \mathrm{glc}}(\mathrm{cell} / \mathrm{mmol})$ & $1.1 \times 10^{8}$ & $2.6 \times 10^{8}$ \\
\hline $\mathrm{Y}_{\mathrm{x}, \mathrm{gln}}(\mathrm{cell} / \mathrm{mmol})$ & $5.6 \times 10^{8}$ & $8 \times 10^{8}$ \\
\hline$\alpha_{1}(\mathrm{mM}$ L/cell-h) & $3.4 \times 10^{-13}$ & $3.4 \times 10^{-13}$ \\
\hline$\alpha_{2}(\mathrm{mM})$ & 4 & 4 \\
\hline$\gamma_{1}(-)$ & $10^{-1}$ & $10^{-1}$ \\
\hline$\gamma_{2}(\mathrm{~h})$ & 2 & 2 \\
\hline$\varepsilon_{1} \quad(-)$ & $9.9 \times 10^{-1}$ & $9.95 \times 10^{-1}$ \\
\hline$\varepsilon_{2}(-)$ & 1 & 1 \\
\hline$\mu_{\max }\left(h^{-1}\right)$ & $5.8 \times 10^{-2}$ & $5.8 \times 10^{-2}$ \\
\hline$\mu_{\mathrm{d}, \max }\left(\mathrm{h}^{-1}\right)$ & $6 \times 10^{-2}$ & $6 \times 10^{-2}$ \\
\hline
\end{tabular}


Table 2. Parameter estimation results for fed-batch culture.

\begin{tabular}{ccccc}
\hline & & \multicolumn{3}{c}{ Confidence Intervals } \\
\hline Parameter & Final value & $90 \%$ & $95 \%$ & $99 \%$ \\
\hline $\mathrm{Y}_{\mathrm{x}, \text { glc }}$ & $2.6 \times 10^{8}$ & $2.224 \times 10^{7}$ & $2.654 \times 10^{7}$ & $3.494 \times 10^{7}$ \\
$\mathrm{Y}_{\mathrm{x}, \text { gln }}$ & $8 \times 10^{8}$ & $2.134 \times 10^{7}$ & $2.546 \times 10^{7}$ & $3.352 \times 10^{7}$ \\
$\mathrm{Y}_{\text {lac,glc }}$ & 2.0 & $1.768 \times 10^{-1}$ & $2.109 \times 10^{-1}$ & $2.777 \times 10^{-1}$ \\
$\mathrm{Y}_{\text {amm, gln }}$ & $4.5 \times 10^{-1}$ & $6.564 \times 10^{-2}$ & $7.832 \times 10^{-2}$ & $1.031 \times 10^{-1}$ \\
$\mathrm{~K}_{\text {lysis }}$ & $3 . \times 10^{-2}$ & $2.520 \times 10^{-3}$ & $3.007 \times 10^{-3}$ & $3.959 \times 10^{-3}$ \\
$\mu_{\max }$ & $5.4 \times 10^{-2}$ & $8.306 \times 10^{-4}$ & $9.909 \times 10^{-4}$ & $1.305 \times 10^{-3}$ \\
$\mathrm{~N}_{\mathrm{H}}$ & 101 & 5.067 & 6.045 & 7.959 \\
\hline
\end{tabular}




\section{FIGURE LEGENDS}

Figure 1. Framework for systematic development of predictive mathematical models for animal cell cultures.

Figure 2. Comparison of model results for extracellular MAb concentration (--) and viable cell concentration $(-)$ with experimental data from batch cultures ( $\circ$ and $\bullet$, respectively).

Figure 3. Comparison of model results for glutamine (-) and ammonia concentrations (- $)$ with experimental data from batch cultures $(\bullet$ and $\circ$, respectively).

Figure 4. Comparison of model results for glucose (-) and lactate concentrations (- $)$ with experimental data from batch cultures $(\bullet$ and $\circ$, respectively).

Figure 5. Comparison of model results for viable (-) and total (--) cell concentrations with experimental data from designed fed-batch cultures $(\bullet$ and $\circ$, respectively).

Figure 6. Comparison of model results for glutamine (-) and ammonia (- - ) concentrations with experimental data from designed fed-batch cultures $(\bullet$ and $\circ$, respectively).

Figure 7. Comparison of model results for extracellular MAb concentration (-) with experimental data from designed fed-batch cultures $(\bullet)$.

Figure 8. Comparison of model results for glutamine (-) and ammonia (- - ) concentrations with experimental data from independent fed-batch cultures $(\bullet$ and $\circ$, respectively).

Figure 9. Comparison of model results for viable (-) and total (--) cell concentrations with experimental data from independent fed-batch cultures $(\bullet$ and $\circ$, respectively).

Figure 10. Comparison of model results for extracellular MAb concentration (-) with experimental data from independent fed-batch cultures $(\bullet)$. 


\section{FIGURES}

\begin{tabular}{|c|c|c|c|}
\hline $\begin{array}{l}\text { Step 1 } \\
\text { Model development* } \\
\text { 1. } \begin{array}{l}\text { Model structure } \\
\text { defined based on }\end{array} \\
\text { - } \quad \begin{array}{l}\text { experimental system } \\
\text { available for } \\
\text { validation }\end{array} \\
\text { - } \begin{array}{l}\text { selected model } \\
\text { application }\end{array} \\
\text { 2. } \begin{array}{l}\text { Physiological ranges } \\
\text { for parameter values }\end{array} \\
\text { established based on } \\
\text { literature data }\end{array}$ & $\begin{array}{l}\text { Step } 2 \\
\text { Model analysis } \\
\text { 1. } \\
\text { - } \quad \text { fonsitivity analysis* qualitative } \\
\text { assessment of } \\
\text { 'sensitive' } \\
\text { parameters } \\
\text { for identification of } \\
\text { most informative } \\
\text { sampling times } \\
\text { Batch experiments for } \\
\text { providing initial } \\
\text { parameter estimates }{ }^{\dagger} \\
\text { *(Kontoravdi et al., 2005) } \\
\text { †This work }\end{array}$ & $\begin{array}{l}\text { Mtep } 3 \\
\text { Model validation } \\
\text { 1. } \\
\begin{array}{l}\text { Optimal design of dynamic } \\
\text { experiments based on } \\
\text { - }\end{array} \\
\text { 'sengeting the estimation of } \\
\text { - } \quad \text { degrees of freedom (feed } \\
\text { volume and time of addition) } \\
\text { 2. Experimentation } \\
\text { 3. Parameter estimation and } \\
\text { model validation } \\
\text { The above steps are repeated until } \\
\text { model exhibits satisfactory } \\
\text { agreement with experimental data } \\
\text { †This work }\end{array}$ & \begin{tabular}{ll}
\multicolumn{1}{c}{ Step 4} \\
Predictive capability \\
1. & Perform an \\
& independent \\
& dynamic experiment \\
2. & Check and confirm \\
model validity and \\
predictive capability \\
by comparing model \\
simulation results \\
with resulting set of \\
data
\end{tabular} \\
\hline
\end{tabular}

Figure 1 


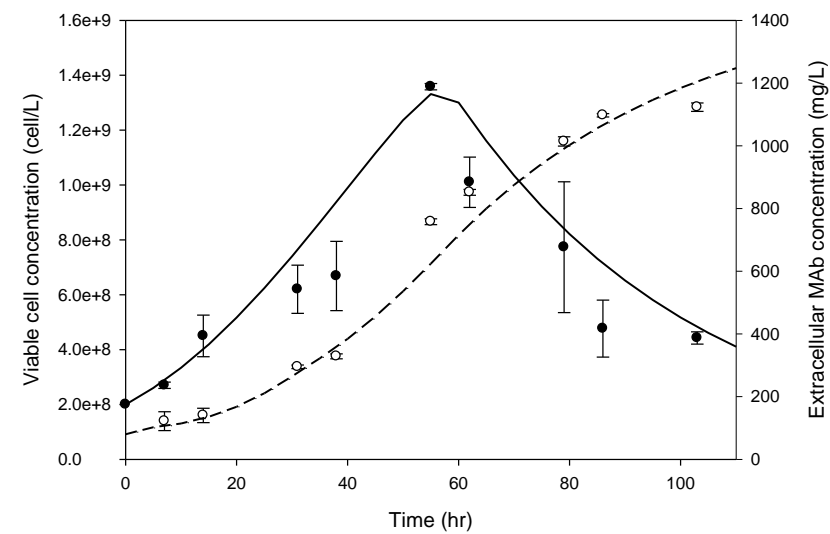

Figure 2 


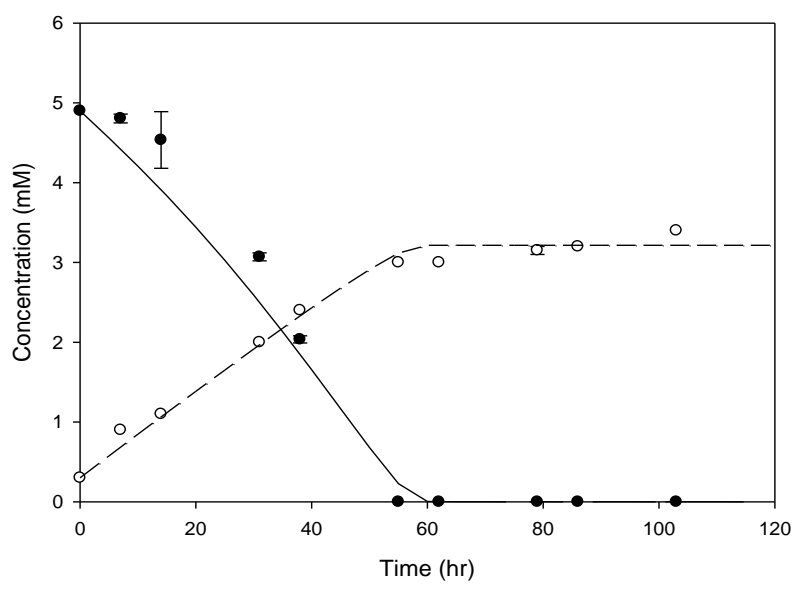

Figure 3 


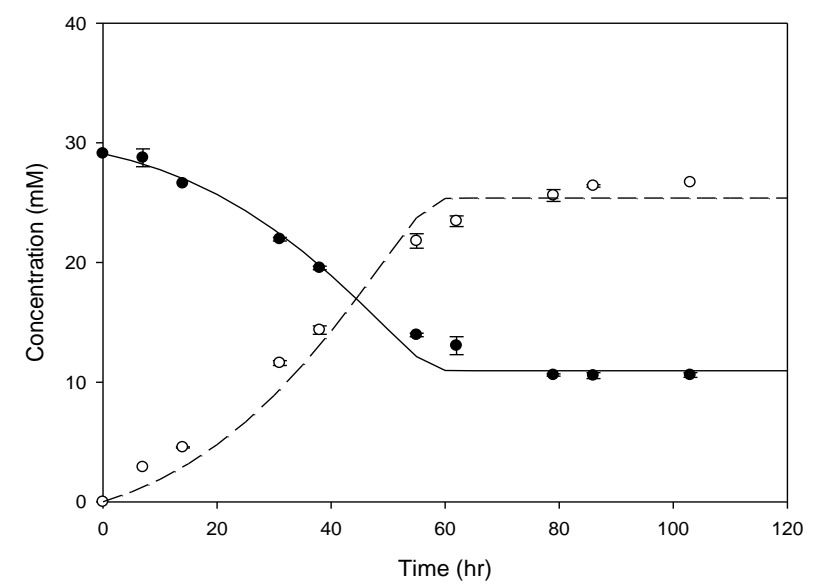

Figure 4 


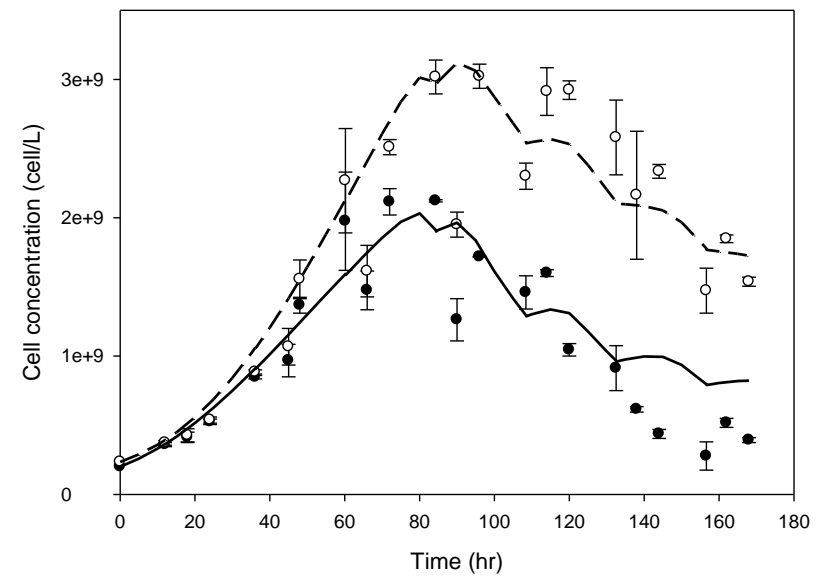

Figure 5 


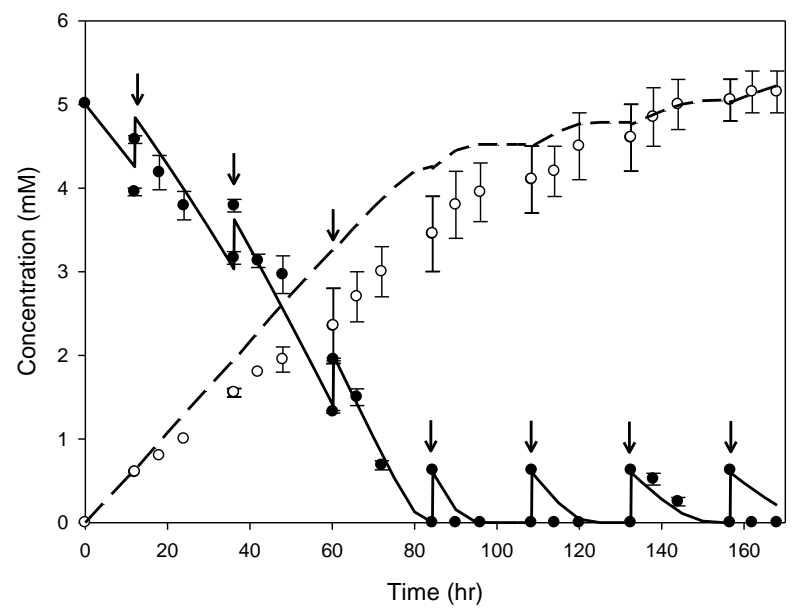

Figure 6 


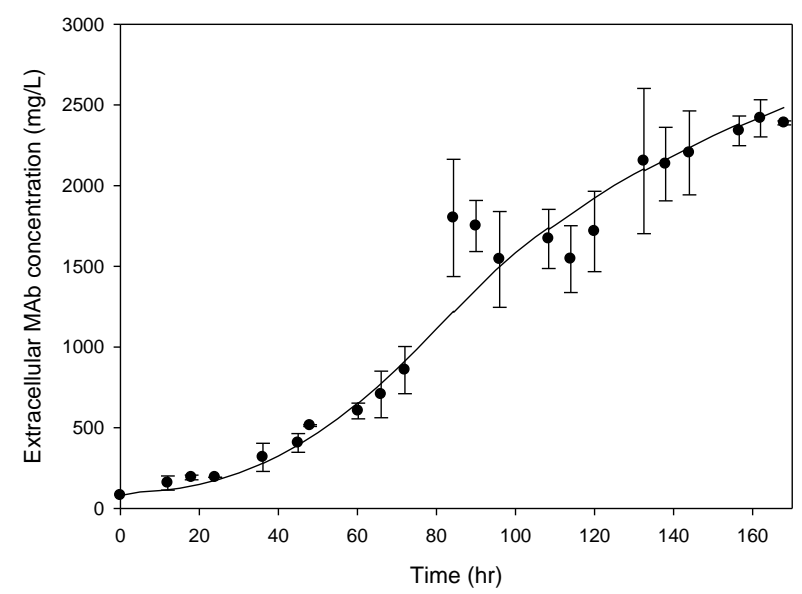

Figure 7 


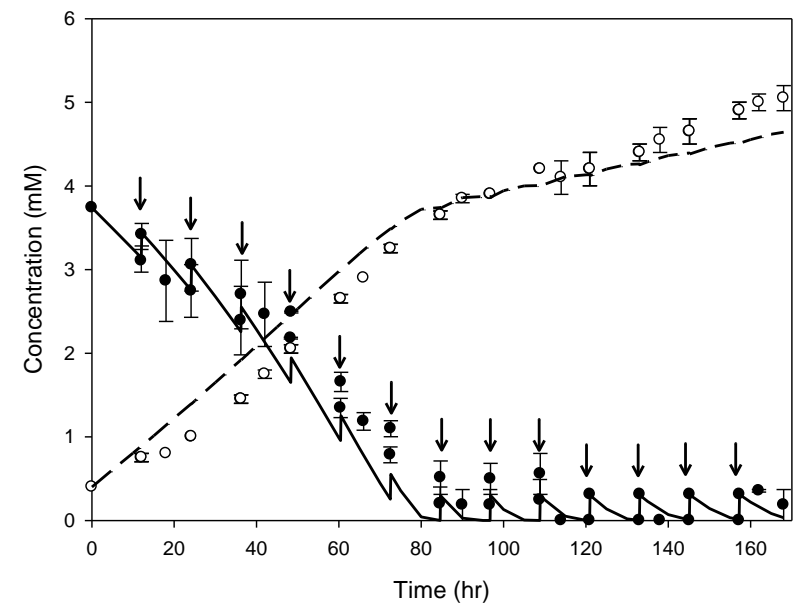

Figure 8 


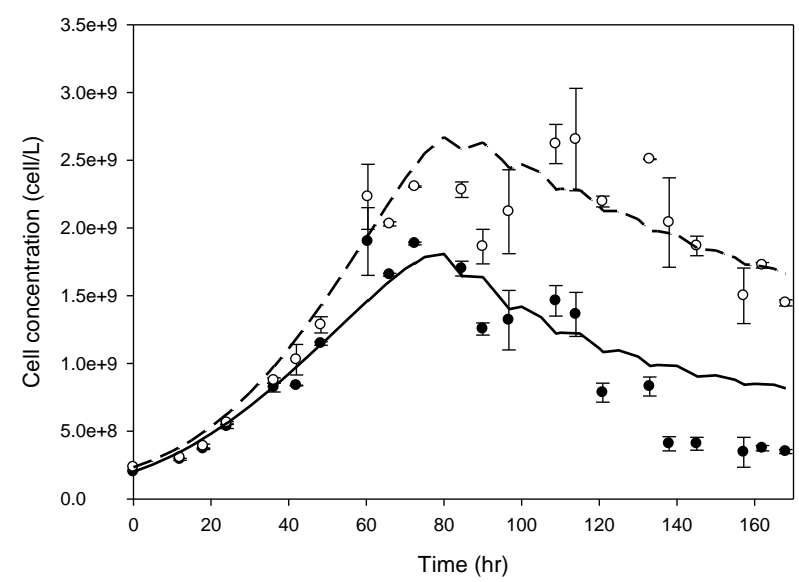

Figure 9 


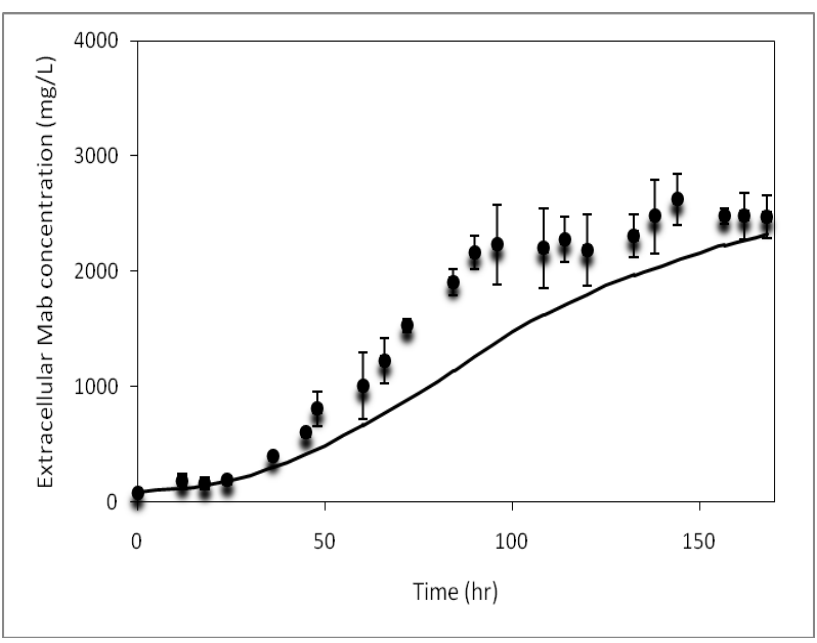

Figure 10 
Appendix A: List of model equations (Kontoravdi et al., 2005).

The mass balances on the viable and total cell populations, based on conventional assumptions such as perfect mixing and negligible dilution effect, are:

$$
\begin{aligned}
& \frac{d V}{d t}=F_{\text {in }}-F_{\text {out }}, \\
& \frac{d\left(V X_{v}\right)}{d t}=\mu V X_{v}-\mu_{d} V X_{v}-F_{\text {out }} X_{v}, \\
& \frac{d\left(V X_{t}\right)}{d t}=\mu V X_{v}-F_{\text {out }} X_{t} .
\end{aligned}
$$

The specific cell growth rate is determined by the concentrations of the glucose, glutamine, lactate and ammonia, following Monod kinetics:

$$
\begin{aligned}
& \mu=\mu_{\max } f_{\lim } f_{i n h}, \\
& f_{\lim }=\left(\frac{[G L C]}{K_{g l c}+[G L C]}\right)\left(\frac{[G L N]}{K_{g \ln }+[G L N]}\right), \\
& f_{\text {inh }}=\left(\frac{K I_{l a c}}{K I_{l a c}+[L A C]}\right)\left(\frac{K I_{a m m}}{K I_{a m m}+[A M M]}\right) .
\end{aligned}
$$

Based on the assumption that cell death only depends on the concentration of ammonia in the extracellular medium, the rate of cell death is determined by:

$$
\mu_{d}=\frac{\mu_{d, \max }}{1+\left(\frac{K_{d, a m m}}{[A M M]}\right)^{n}}, \mathrm{n}>1 .
$$

The mass balances on glucose and glutamine around the bioreactor are:

$$
\begin{aligned}
& \frac{d(V[G L C])}{d t}=-Q_{g l c} V X_{v}+F_{\text {in }}[G L C]_{i n}-F_{\text {out }}[G L C], \\
& Q_{g l c}=\frac{\mu}{Y_{x, g l c}}+m_{g l c}, \\
& \frac{d(V[G L N])}{d t}=-Q_{g \ln } V X_{v}-K_{d g \ln } V[G L N]+F_{\text {in }}[G L N]_{\text {in }}-F_{\text {out }}[G L N], \\
& Q_{g \ln }=\frac{\mu}{Y_{x, g \ln }}+m_{g \ln }, \\
& m_{g \ln }=\frac{\alpha_{1}[G L N]}{\alpha_{2}+[G L N]} .
\end{aligned}
$$

The mass balances for lactate and ammonia are:

$$
\begin{aligned}
& \frac{d(V[L A C])}{d t}=Q_{l a c} V X_{v}-F_{\text {out }}[L A C], \\
& Q_{l a c}=Y_{l a c, g l c} Q_{g l c}, \\
& \frac{d(V[A M M])}{d t}=Q_{a m m} V X_{v}+K_{d g \ln } V[G L N]-F_{\text {out }}[A M M], \\
& Q_{a m m}=Y_{a m m, g \ln } Q_{g \ln } .
\end{aligned}
$$

The heavy- and light-chain mRNA balances are:

$\frac{d m_{H}}{d t}=N_{H} S_{H}-K m_{H}$, 
$\frac{d m_{L}}{d t}=N_{L} S_{L}-K m_{L}$

The intraER heavy- and light-chain and assembly intermediates balances are:

$\frac{d[H]}{d t}=T_{H} m_{H}-R_{H}$

$\frac{d[L]}{d t}=T_{L} m_{L}-R_{L}$

where,

$R_{H}=\frac{2}{3} K_{A}[H]^{2}$

$R_{L}=2 K_{A}\left[H_{2}\right]\left[L^{\prime}\right]+K_{A}\left[H_{2} L\right][L]$,

and

$\frac{d\left[H_{2}\right]}{d t}=\frac{1}{3} K_{A}[H]^{2}-2 K_{A}\left[H_{2}\right][L]$

$\frac{\left.d \llbracket H_{2} L\right]}{d t}=2 K_{A}\left[H_{2} \rrbracket[L]-K_{A}\left[H_{2} L \rrbracket L\right]\right.$

The intraER MAb balance is:

$\frac{d\left[H_{2} L_{2}\right]_{E R}}{d t}=K_{A}\left[H_{2} L\right][L]-K_{E R}\left[H_{2} L_{2}\right]_{E R}$.

Similarly, for the Golgi apparatus:

$\frac{d\left[H_{2} L_{2}\right]_{G}}{d t}=\varepsilon_{1} K_{E R}\left[H_{2} L_{2}\right]_{E R}-K_{G}\left[H_{2} L_{2}\right]_{G}$.

Finally, the rate of MAb production is:

$\frac{d(V[M A b])}{d t}=\left(\gamma_{2}-\gamma_{1} \mu\right) Q_{M A b} V X_{v}-F_{\text {out }}[M A b]$

$Q_{M A b}=\varepsilon_{2} \lambda K_{G}\left[H_{2} L_{2}\right]_{G}$. 